\title{
Statistical properties of magnetic structures: Their dependence on scale and solar activity
}

\author{
N. Meunier ${ }^{\star}$ \\ Observatoire Midi-Pyrénées, 57 Avenue d'Azereix, BP 826, 65008 Tarbes Cedex, France \\ Received 27 November 2002 / Accepted 24 April 2003

\begin{abstract}
A long data set of MDI/SOHO full-disk magnetograms is analyzed in order to provide a large sample of network features as well as active regions associated with their magnetic properties (magnetic flux and magnetic flux density for example). The main objective of the paper is to study the variations of these properties along the solar cycle, and to compare the behavior of the magnetic features at various scales (covering three orders of magnitude in size). I also investigate the dependence of these properties on the activity level in their neighborhood and in the context of their global configuration on the solar disk. This study is important for a better understanding of the emergence and decay of active regions, as well as of the role of the network in the removal of the magnetic flux and in solar irradiance variations. The main results are as follows. (i) I observe a characteristic scale in the range $400-800 \mathrm{Mm}^{2}$ (close to the supergranular scale) below which the size and magnetic flux distributions are power laws down to $40 \mathrm{Mm}^{2}$ (corresponding to the range $3 \times 10^{19}-10^{21} \mathrm{Mx}$ ). This scale increases from solar minimum to solar maximum. The shape of the distributions also suggests a strong similarity between small active regions (below the supergranular scale) and network patches. (ii) The North-South asymmetry in the number of structures, compatible with easier-to-detect leading structures in most cases, surprisingly exhibits a sign reversal for the weakest structures, suggesting a different origin, while the East-West asymmetry is compatible with a predominantly eastward inclination of magnetic flux tubes, including network structures and not only for emerging active regions as suggested by models. (iii) The dependence of the magnetic characterictics on the size of the structures is found to be variable with the activity level, the magnetic flux beeing more concentrated at solar maximum (especially for small features). Small-scale properties are also dependent on the proximity of an active region. (iv) Latitudinal distributions and flux variations along the solar cycle suggests a significant role of the meridional flows in the properties of the very small network features as well as an influence of supergranulation properties. (v) The variations of the magnetic properties of network elements and active regions with time are strongly size-dependent. The results also show a correlation between the number (or magnetic flux) of network structures and the sunspot cycle, with an amplitude larger than expected from previous results.
\end{abstract}

Key words. Sun: magnetic fields - Sun: photosphere - Sun: activity - Sun: faculae, plages

\section{Introduction}

Most of the magnetic network flux emerges as ephemeral regions and covers the whole surface of the Sun outside active regions. However, these small flux concentrations are organized in a large-scale pattern resulting from the decay of active regions: The patches of small flux tubes constituting the network are mostly present at the edge of supergranular cells $(\sim 30000 \mathrm{~km}$ in diameter), also observed as velocity patterns in dopplergrams and from granule dynamics. At first order, the individual flux tubes are similar to the flux tubes in plages. With a diameter smaller than $300 \mathrm{~km}$, they are mostly vertical, with magnetic fields larger than $1 \mathrm{kG}$ and are observed as bright structures warmer than their surrounding.

The understanding of the small and large scale properties of the network allows to comprehend better the processes

\footnotetext{
* e-mail: meunier@bagn.obs-mip.fr
}

leading to the diffusion of magnetic flux away from active regions and to the removal of the magnetic flux from the surface of the Sun. Such an analysis is therefore of great interest in the context of the study of the emergence and decay of active regions as well as ephemeral regions. It should supply information on the processes at the origin of the concentration of the magnetic flux in network flux tubes and its interaction with the dynamics of the photosphere at various scales. On the small scale end of magnetic strutures, it should also provide some clues about the origin of intranetwork magnetic fields, weak field structures that are not well understood yet. Furthermore, the contribution of the network to solar irradiance variations has long been a controversial subject.

Network properties have often been studied through the diffusion coefficient (see for example Schrijver et al. 1996 and references therein), but no work on its variation along the solar cycle has been done. On the other hand, the work of 
Harvey (1993) on bipolar regions (from ephemeral regions to large active regions) considers properties such as lifetime or tilt (among others) but most magnetic characteristics such as the flux density are not analyzed (only the flux content of the regions have been studied, and not in detail). Structures not involved in a bipolar configuration (such as network features) are not included in this study. Therefore the intrinsic properties of the magnetic network are mostly known through a few spectropolarimetric studies (for example Sànchez Almeida \& Lites 2000; Sigwarth 2001; Socas-Navarro \& Sànchez Almeida 2002) of a small number of structures without any simultaneous large-scale analysis. On the other hand, several studies of the network contribution to solar irradiance variations have been performed over the last two decades. White \& Livingston (1981) found the Ca II K emission in quiet regions at the equator to be constant during the solar cycle. Labonte \& Howard (1982) studying magnetic fluxes found no cycle dependence of the network magnetic flux either. On the other hand, Muller \& Roudier $(1984,1994)$ found the number of network bright points (hereafter NBP) in very quiet regions to be anticorrelated with the solar cycle. Later however, Foukal \& Lean (1988) showed that the network contribution is necessary to explain the general downturn of the solar irradiance during the declining phase of the solar cycle. Foukal et al. (1991) showed that the quiet sun network covers $15 \%$ and $21 \%$ of the solar surface at solar minimum and solar maximum (ratio of 1.5) respectively, and the network properties are consistent with the solar irradiance variations. Nishikawa (1994) and more recently Lean et al. (1998) confirmed the contribution of the network to solar irradiance variations.

There is therefore a need for more detailed studies of possible cyclic variation of the magnetic properties of the network, as well as the dependence of their properties on their magnetic environment (activity level and relationship with active regions). Such approaches should include a very large sample of network features for a good statistical study. Also, the puzzling anticorrelation with the solar cycle obtained by Muller \& Roudier $(1984,1994)$ requires an explanation in view of the more recent results about the network contribution to solar irradiance variations. These studies about irradiance variations have been made considering constant properties of the network features throughout the solar cycle, which may not be correct.

The objective of this paper is to study the properties of network features as well as their variation along the solar cycle, and to compare them with active regions. I also investigate the variation of network properties in the context of their global configuration on the solar disk. For this purpose, a long data set of MDI full disk magnetograms (Sect. 2) is analyzed in order to study the characteristics of magnetic structures over more than three orders of magnitude in size from network to active regions (Sect. 3). The evolution of these characteristics along the solar cycle (6 years from minimum to maximum) is studied in Sect. 4. Section 5 presents a summary of the results, which are discussed in Sect. 6. Very recently, a study focusing on bipolar regions using the same data set but a slightly different approach has been published by Hagenaar et al. (2003).

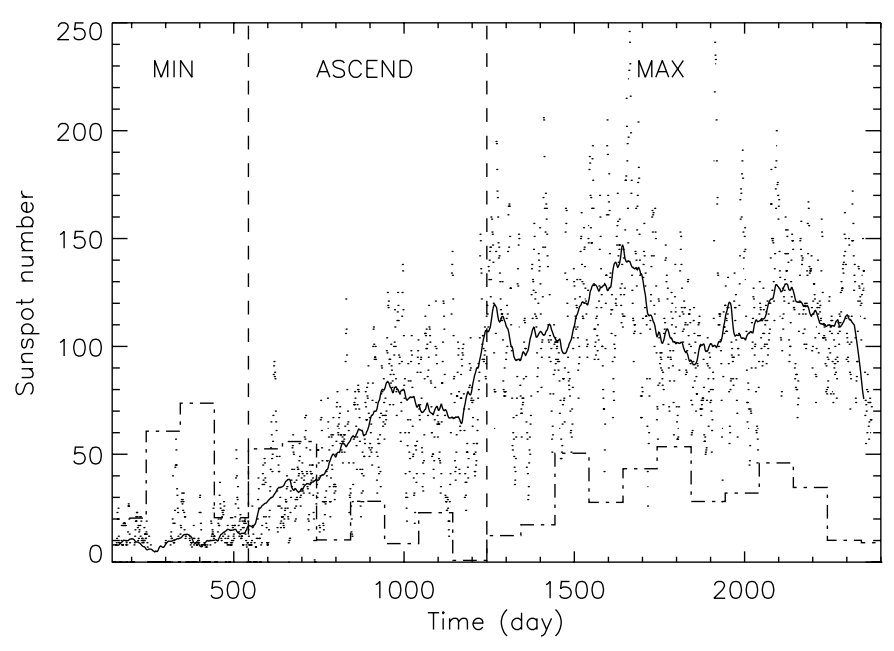

Fig. 1. Daily sunspot number (dots) versus time (day 1 is Jan. 1996, 1). The solid line represents the sunspot number smoothed over 100 days. The dashed-dotted line is the number of processed magnetograms divided by 10 for each 100 day period (starting on May 1996, 23).

\section{Observations and data processing}

\subsection{MDI/SOHO: Full-disk magnetograms}

MDI/SOHO observations (Scherrer et al. 1995) provide maps of the line-of-sight component of the magnetic field averaged over the resolution element $(2 \operatorname{arcsec})$. The observations are made in the Ni I $6768 \AA$ spectral line. I select 5-min full-disk magnetograms only, because they are less noisy (noise level of $\sim 8 \mathrm{G}$, see for example Meunier 1999a). This led to a data set of 7184 images between May 1996, 23 and June 2002, 19, covering 6 years. These magnetograms are separated by a multiple of $96 \mathrm{~min}$.

Figure 1 shows the Wolf number and data availability (number of magnetograms per 100 day period) during that period. The data availability is poor at the end of the ascending phase. In order to study solar cycle variations, I define the three following periods, illustrated in Fig. 1: The cycle minimum from day 143 (May 23, 1996) until day 543 (June 1997, 27), an ascending phase from day 543 until day 1243 (May 1999, 28) and the cycle maximum after that date until the end of the data set. Solar variations will be subsequently studied either over these three periods or over the 23 100-day period constituting the data set.

I also study 15 series of one-hour data sets (60 magnetograms each with a temporal cadence of $1 \mathrm{~min}$ ) in order to analyze the variation of the noise level with time in Sect. 2.3.

\subsection{Data processing}

Magnetograms are analyzed using a method similar to that described in Meunier (1999a), except that we consider $B / \mu$ (where $\mu=\cos \theta, \theta$ being the angle from disk center and $B$ is the line-of-sight magnetic field provided by MDI) instead of $B$, as is done in Hagenaar (2001) for ephemeral regions, because the magnetic field lines in both active regions and network regions are believed to be close to the vertical, within $10^{\circ}$ (the only exception is the penumbra of sunspots but this 
Table 1. Number of structures of all sizes for the different magnetic thresholds.

\begin{tabular}{ccc}
\hline \hline Magnetic threshold & Number & $<$ Number $>$ per image \\
\hline $25 \mathrm{G}$ & 6967532 & 970 \\
$40 \mathrm{G}$ & 4291752 & 597 \\
\hline
\end{tabular}

concerns only a very small proportion of the studied features). Computations using $B$ instead of $B / \mu$ do not differ significantly from the results presented in this paper however. Each structure above a given magnetic threshold (discussed below) is determined within $53^{\circ}$ from disk center $\left(r<0.8 R_{\odot}\right)$. Areas $A$ and perimeters $P$ are corrected for projection effects. They are converted to $\mathrm{Mm}^{2}$ and to $\mathrm{Mm}$ respectively. Structures of different polarities are counted separately (the threshold is applied to the signed magnetograms). In addition, I compute the following parameters in order to characterize the magnetic properties of the structures: ( $i$ ) The magnetic flux per structure, $\Phi$ (in $M x$ ) (the unsigned value is often used in the paper); (ii) The maximum unsigned magnetic field in the structure, $\left|B_{m}\right|$ (this is also a flux density averaged over the MDI pixel, not an intrinsic field strength); (iii) The ratio between the maximum magnetic field $B_{m}$ and the average magnetic field in the structure $\bar{B} ;(i v)$ And finally $|\Phi|$ divided by the area, which provides the unsigned magnetic flux per "pixel" in the structure, where the pixel size corresponds to that of disk center (this is the mean magnetic field density in the structure), or flux density. In order to characterize the activity level in the close neighborhood of a structure, I also compute the number of pixels over the magnetic threshold in the range of $[R+20, R+40]$ pixels (where $R$ is defined as $\sqrt{A / \pi}$ ).

The number of structures per image for the two thresholds ( $25 \mathrm{G}$ and $40 \mathrm{G}$ ) is shown in Table 1 . These thresholds correspond to 3 and 5 times the noise level in these magnetograms, respectively (see Sect. 2.3). An example of a magnetogram after the application of these thresholds is shown in Fig. 2 for $r<0.8 R_{\odot}$. The threshold of $25 \mathrm{G}$ is a minimum considering the noise level. The choice of a second threshold allows to get more informations. A low threshold allows to look at weaker flux features compared to a larger threshold, although it could be more sensitive to the noise. We will see that most of the results are not strongly dependent on this choice.

\subsection{Limitations}

\subsubsection{Sensitivity limitations}

In this study, structures with a size below 4 pixels are eliminated because of the limited spatial resolution. Note that the small features observed in the south-western quadrant of the $25 \mathrm{G}$ thresholded magnetogram in Fig. 2 (due to a higher noise level) are removed when selecting these sizes only. In addition to the limit on the size of the structures, the magnetic thresholds impose a limit on the magnetic flux density, which could correspond to different types of structures (for example structures larger than 4 pixels but with a weak magnetic flux everywhere).
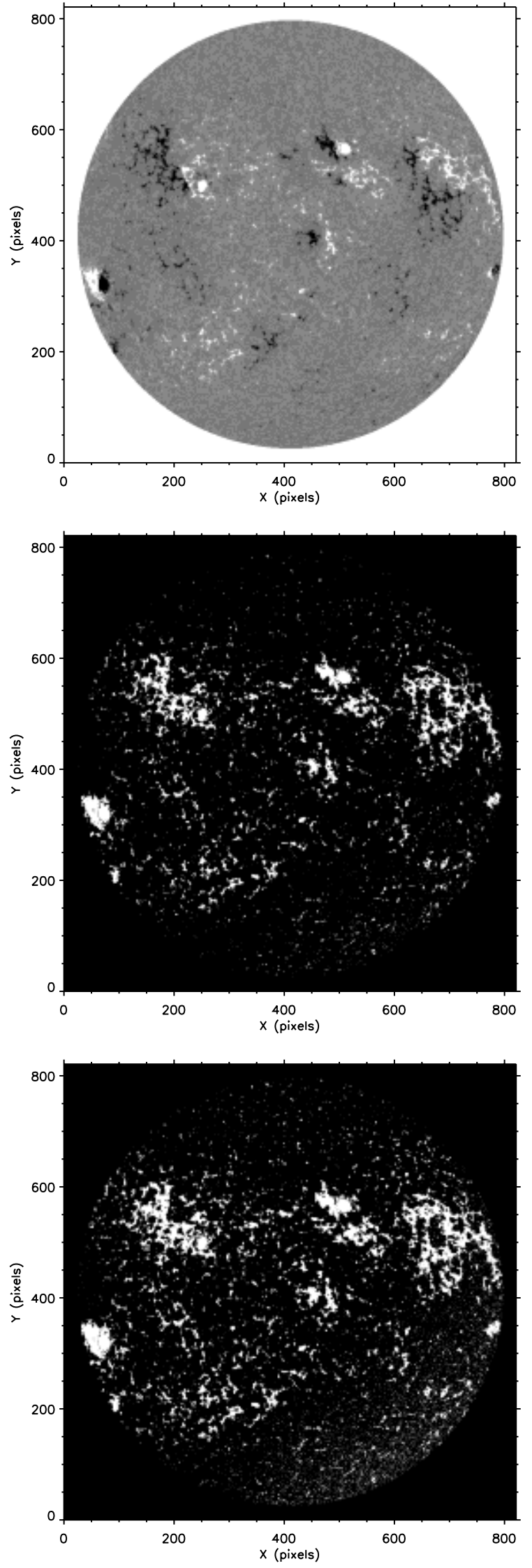

Fig. 2. Top: a magnetogram for $r<0.8 R_{\odot}$. Middle: after a $40 \mathrm{G}$ threshold. Bottom: after a $25 \mathrm{G}$ threshold. 
One should also remember that the magnetograms provide only the line-of-sight magnetic flux over the resolution element.

\subsubsection{Influence of the noise}

In order to check the noise level and its variation with time, I study 15 one-hour data sets of full-disk magnetograms spread over the 6 years. We process each data set according to the following steps: (i) Pairs of 5-min magnetograms are computed using 10 images separated by 1 min; (ii) Pixels with magnetic fields larger than $25 \mathrm{G}$ in the 5-min magnetograms are removed in order to consider quiet regions only; (iii) The difference between magnetograms of each pair is computed; (iv) The percentage of pixels above the magnetic threshold for $r<0.8 R_{\odot}$ as well as the width of the corresponding distribution of magnetic fields are computed.

The results confirm the $8 \mathrm{G}$ noise level (the average over the whole data set is $8.7 \mathrm{G}$ ). On average, the percentage of pixels in the magnetogram differences above the $40 \mathrm{G}$ threshold is $0.03 \%$, and $0.9 \%$ above the $25 \mathrm{G}$ threshold. The noise level seems to increase slightly over the 6 years of the study: from a linear fit on the noise versus time, the noise level is around $8.2 \mathrm{G}$ at the beginning of the period and around $9.1 \mathrm{G}$ at the end of the period. A similar variation is observed in all hemispheres although the average level is different: $8.4 \mathrm{G}$ in the North, $9.1 \mathrm{G}$ in the South, 8.2 G in the East and 9.3 G in the West. Simulations made by adding noise of different amplitudes (typical of the 4 hemispheres) are made in order to study their influence on the results. The only significant influence concerns the number of structures and size distribution (Sect. 3.1) and will be discussed later. The other characteristics (such as the dependence of the magnetic flux on the size) exhibits variations due to different noise levels that are insignificant. It is also important to note that the noise measured here contains not only instrumental noise but also some "noise" of solar origin which is due to weak fields or even strong fields with a significant evolution over a few minutes: therefore the actual variation of the instrumental noise is likely to be smaller.

\subsubsection{Non-linearity of MDI magnetic field measurements}

Berger \& Lites (2003) showed that the MDI response concerning magnetic field measurements was starting to be nonlinear above $1600 \mathrm{G}$, with a strong saturation around $2200 \mathrm{G}$. This problem will mostly affect the maximum magnetic field computed in very large regions and, to a lesser extent, the magnetic flux and flux density. Furthermore, even in large structures, the pixels with a magnetic field larger than $1600 \mathrm{G}$ represent the tail of the magnetic field distribution in the structure. It will not affect the thresholding. Small scale feature properties, such as network patches and small active regions, for which no pixel shows a magnetic field above $1600 \mathrm{G}$ are not influenced. Therefore most of the structures we will be interested in are not affected. Note that the values of the measured magnetic field (for example the threshold of $40 \mathrm{G}$ ) is also instrument-dependent, as emphasized by

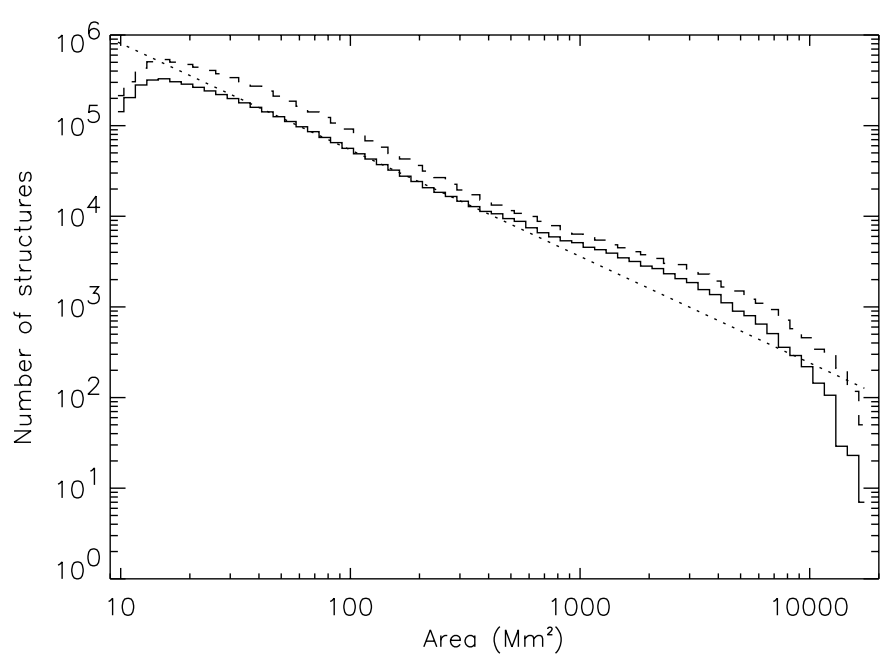

Fig. 3. Size distribution for all structures (solid: $40 \mathrm{G}$ threshold, dashed: $25 \mathrm{G}$ threshold). The dotted line is a linear fit to the $40 \mathrm{G}$ distribution in the $40-400 \mathrm{Mm}^{2}$ range on a log-log plot (power law).

Berger \& Lites (2003). They show for example that MDI magnetic fields are underestimated by at least $30 \%$.

\section{Magnetic feature characteristics}

\subsection{Size and asymmetries}

\subsubsection{Size distribution}

Figure 3 shows the size distribution on a $\log / \log$ plot. It is important to point out that a direct comparison with size distributions obtained for active regions by other authors (Harvey \& Zwaan 1993, for example) is difficult because they have counted each active region region once, taking into account their lifetime. This is not the case here: when dealing with small magnetic features (such as in this study), this is a lot more complex to perform and would request a better temporal cadence. This is beyond the scope of this paper and therefore a feature or region is counted each time it is present on the disk. As a consequence, the distributions shown in this paper are not directly related to the emergence rate of regions but correspond to the proportion of features of different sizes present on the disk at a given time. The lifetime being dependent on the size, we expect a slope smaller than the one found by Harvey \& Zwaan for example.

The size distribution exhibits a maximum around $15 \mathrm{Mm}^{2}$, for both thresholds ( $25 \mathrm{G}$ and $40 \mathrm{G}$ ). This may be due to the fact that the smaller features may not be visible at that magnetic threshold. This has also been observed by Schrijver et al. (1997) for small bipoles (ephemeral regions). The distribution is then a power law in the range $40 \mathrm{Mm}^{2}$ (linear size of $7100 \mathrm{~km}$ ) to $400 \mathrm{Mm}^{2}$ (linear size of $25000 \mathrm{~km}$ ), although the departure from the power law is mostly observed above $800 \mathrm{Mm}^{2}$ (linear size of $31900 \mathrm{~km}$, close to the supergranulation scale). Note that this supergranular cell size is much larger than network features: Wang (1988), using cross-correlation of magnetograms, finds a typical size of the network of $5700 \mathrm{~km}$ $\left(33 \mathrm{Mm}^{2}\right)$ with a maximum size of $\sim 80 \mathrm{Mm}^{2}$. Above $800 \mathrm{Mm}^{2}$, 
the distribution is slightly increasing and then dropping. This suggests that properties are similar from network to small active regions, while large active regions show a different behavior. This is in agreement with Garcia de la Rosa (1983), who observed that small active regions below $10^{21} \mathrm{Mx}$ were related to the decay of larger active regions: We will see in the next section that this flux corresponds to the supergranular size regions seen here. A shift along the $A$ axis of the $25 \mathrm{G}$ threshold curve to match the $40 \mathrm{G}$ threshold curve shows a very good agreement for $A$ larger than $\sim 100 \mathrm{Mm}^{2}$. Below this scale, the $25 \mathrm{G}$ distribution is starting to show some structures that are not seen on the $40 \mathrm{G}$ distribution. The three domains observed here will often be used in the paper to compare the behavior of structures of different sizes.

\subsubsection{North-South asymmetry}

North-South asymmetries are listed in Table 2. At the $40 \mathrm{G}$ threshold, there are more structures in the northern hemisphere for regions of all sizes, although the amplitude of the asymmetry is larger for sizes in the range $40-800 \mathrm{Mm}^{2}$. At the $25 \mathrm{G}$ threshold, the situation is surprisingly reversed when considering all sizes: This is due to a larger number of small structures (below $40 \mathrm{Mm}^{2}$ ) in the southern hemisphere when using this threshold. One should remember that the sample of structures when going from a $40 \mathrm{G}$ threshold to a $25 \mathrm{G}$ threshold is different: Many structures in the range $10-40 \mathrm{Mm}^{2}$ at the $40 \mathrm{G}$ threshold will be above $40 \mathrm{Mm}^{2}$ at the $25 \mathrm{G}$ threshold, while the range $10-40 \mathrm{Mm}^{2}$ at $25 \mathrm{G}$ threshold will contain many new structures corresponding to weaker fluxes that were excluded from the $40 \mathrm{G}$ sample. So this result suggests that the asymmetry is actually reversing for weak flux structures which are not seen at the $40 \mathrm{G}$ threshold, as if they were due to a different process.

The amplitude of the asymmetry must be compared to the noise influence in the different hemispheres. Simulations show that the different noise levels in the northern and southern hemispheres (Sect. 2.3.2) should lead to $0.4 \%$ more structures in the South, with a more pronounced effect for small structures $\left(0.8 \%\right.$ for sizes below $\left.25 \mathrm{Mm}^{2}\right)$. Therefore the positive asymmetry observed here (more structures in the North) are likely to be underestimated and the sign reversal is probably true.

\subsubsection{Polarity asymmetry}

The two polarities also exhibit different behaviors. At the $40 \mathrm{G}$ threshold, there are more structures of negative polarity. However this is true for small structures only (below $40 \mathrm{Mm}^{2}$ ) while the asymmetry seems to be reversed for larger structures. On the other hand, the North-South asymmetry is quite large when one considers the polarities separately: there is a much larger number of positive polarity structures in the northern hemisphere and of negative polarity structures in the southern hemisphere, i.e. the leading polarity is dominating in each hemisphere ${ }^{1}$. The magnetic flux is known to be more

\footnotetext{
${ }^{1}$ For this cycle, the leading polarity in the northern hemisphere is positive.
}

Table 2. North-South, East-West and polarity asymmetry in the number of structures $(\%)$ for various selections in term of size, polarity and hemisphere (defined as the difference in numbers $N$ of structures - first criterion minus the second one - divided by their sum). The uncertainties correspond to $1 / \sqrt{N}$. The area $A$ is in $\mathrm{Mm}^{2}$.

\begin{tabular}{ccc}
\hline \hline Selection & $25 \mathrm{G}(\%)$ & $40 \mathrm{G}(\%)$ \\
\hline North, South & $-0.43 \pm 0.05$ & $0.98 \pm 0.07$ \\
North, South $(A>800)$ & $2.07 \pm 0.52$ & $0.61 \pm 0.61$ \\
North, South $(40<A<800)$ & $2.07 \pm 0.10$ & $3.03 \pm 0.13$ \\
North, South $(A<40)$ & $-1.48 \pm 0.06$ & $0.15 \pm 0.08$ \\
\hline$B>0, B<0$ & $-2.96 \pm 0.05$ & $-0.61 \pm 0.07$ \\
$B>0, B<0(A>800)$ & $-0.95 \pm 0.53$ & $0.20 \pm 0.61$ \\
$B>0, B<0(40<A<800)$ & $-1.83 \pm 0.10$ & $0.27 \pm 0.13$ \\
$B>0, B<0(A<40)$ & $-3.44 \pm 0.06$ & $-0.99 \pm 0.08$ \\
\hline North, South $(B>0)$ & $3.99 \pm 0.07$ & $5.63 \pm 0.09$ \\
North, South $(B<0)$ & $-4.60 \pm 0.08$ & $-3.61 \pm 0.10$ \\
\hline East, West & $-3.23 \pm 0.05$ & $-2.28 \pm 0.07$ \\
East, West $(B>0$, North $)$ & $-3.67 \pm 0.10$ & $-3.28 \pm 0.13$ \\
East, West $(B<0$, North $)$ & $-1.55 \pm 0.11$ & $-1.84 \pm 0.14$ \\
\hline
\end{tabular}

concentrated in the leading polarity so this would change the detection level for each of them and could explain the result. The asymmetry is also observed for the total fluxes. At the $25 \mathrm{G}$ threshold, there are more negative polarity structures for all sizes, and the asymmetry is of larger amplitude than at the $40 \mathrm{G}$ threshold. The origin of such a difference (especially for large regions) and of this global imbalance is not understood: for most structures, the observed North-South asymmetry and the easier detection of leading polarity should lead to more positive polarity structures, which is not what is observed at the $25 \mathrm{G}$ threshold. The North-South asymmetry is however similar to the one found for the $40 \mathrm{G}$ threshold when separating the polarities.

\subsubsection{East-West asymmetry}

East-West asymmetries for a few selections of parameters are also shown in Table 2. There are always more structures on the western side, even for large structures and this is independent of the polarity: The asymmetry is in the range of 1.8-3.3\% with the $40 \mathrm{G}$ threshold (1.5-4.0\% with the $25 \mathrm{G}$ threshold) for various selections. The amplitude of the asymmetry is larger for the leading polarity (positive in the north for example) than for the following polarity (negative in the north for example). Therefore the behavior is very different from the North-South asymmetry.

The East-West asymmetry could have been due to the noise level, which is known to be larger in the south-western quadrant of MDI magnetograms, especially close to the limb. However this should not influence significantly the larger structures. A simulation shows that we expect $0.6 \%$ more structures in the western hemisphere due to the different noise level, and $1.3 \%$ more structures for small sizes below $25 \mathrm{Mm}^{2}$. Therefore the asymmetry observed here seems significant although slightly overestimated. It is therefore more likely to be due to another effect, for example a different projection effect if leading and following polarities have different typical inclinations with 

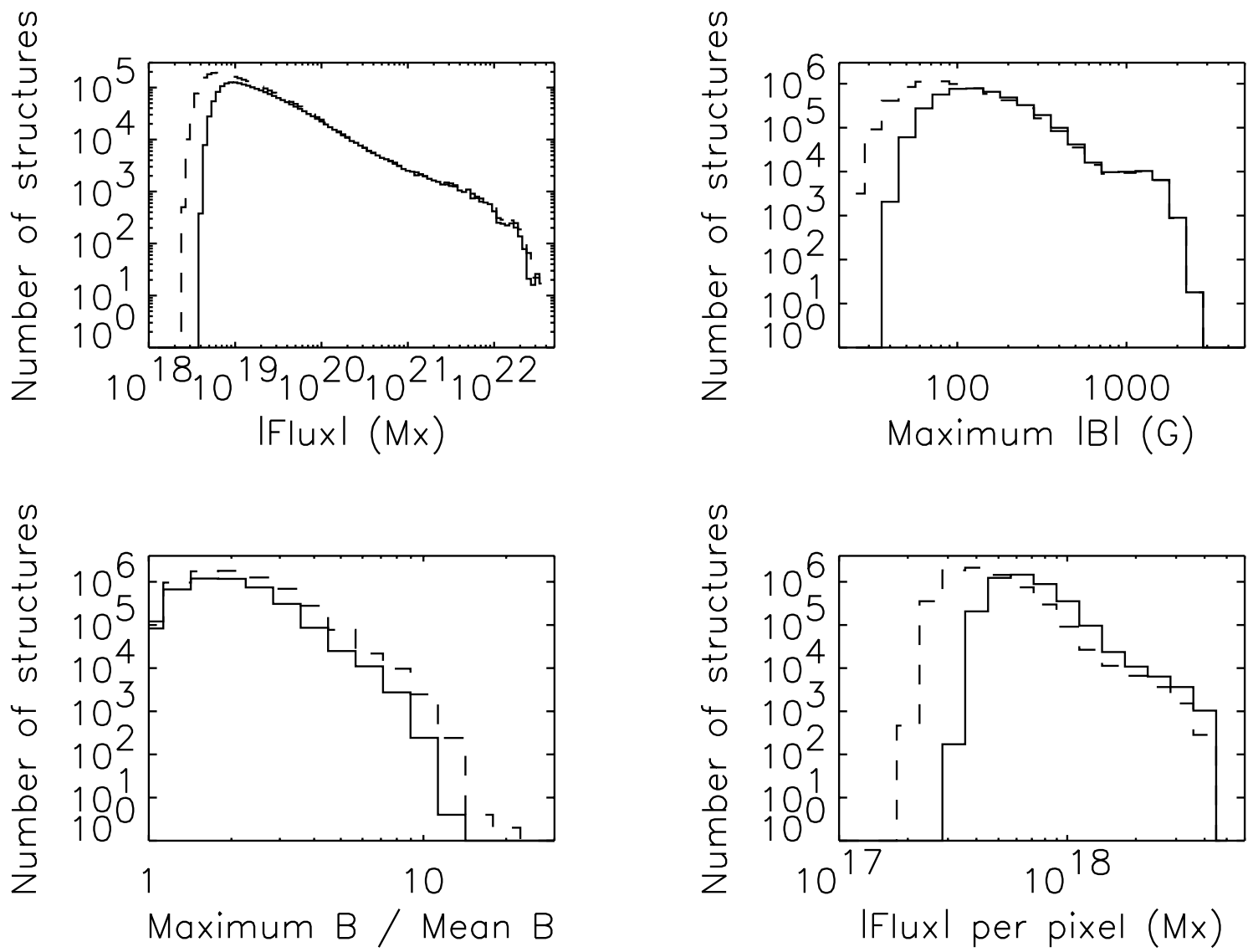

Fig. 4. Distribution of $|\Phi|$ (top left), $\left|B_{m}\right|$ (top right), $B_{m} / \bar{B}$ (bottom left) and $|\Phi| / A$ (bottom right) in all structures (Solid: $40 \mathrm{G}$ threshold, dashed: $25 \mathrm{G}$ threshold).

respect to the vertical for example: the larger number of structures in the West is compatible with a preferentially eastward inclination of the flux tubes that would lead to a different detection level in the two hemispheres. This subject is still controversial. An eastward inclination of the emerging flux tubes has been proposed by van Driel-Gesztelyi \& Petrovay (1990) in order to explain the asymmetry in the proper motions of bipoles. Howard (1991) found an average eastward inclination of $1.9^{\circ}$ of flux tubes in young active regions (inclination that became westward for growing regions) while Harvey (1993) found an westward inclination of ephemeral region flux tubes (using a counting method similar to ours). On the other hand, models predict an eastward inclination (Caligari et al. 1995; Abbett et al. 2001) in agreement with van Driel-Gesztelyi \& Petrovay's predictions and Howard's results for emerging active regions. So our results are compatible with the observations of bipolar active region proper motions and from models, but it is extended to structures of all sizes, including network features.

\subsection{Magnetic field characteristics}

\subsubsection{Magnetic fields of the structures}

In this section I study the magnetic field characteristics of the structures, using the four parameters defined in Sect. 2.2: $|\Phi|$, $\left|B_{m}\right|, B_{m} / \bar{B}$ and $|\Phi| / A$. Figure 4 shows the distribution of these four parameters for both magnetic thresholds (25 and $40 \mathrm{G})$. They are quite similar, although it is of course possible to access smaller values of $|\Phi|$ and $\left|B_{m}\right|$ at $25 \mathrm{G}$, while the ratio between maximum and average magnetic field can reach larger values. Figure 5 shows these four parameters versus the size of the structures. They are all correlated with the size. Linear fits to the parameters versus size (on a $\log / \log$ scale) after averaging over small ranges of size $(\triangle \log A=0.1)$ are also computed. The slopes are indicated in Table 3: This tool will be usefull when looking at solar cycle variations.

The shape of the flux distribution is very similar to that of the size distribution shown in Fig. 3. I observe a modification in behavior of the flux distribution around $10^{21} \mathrm{Mx}$, as expected given the observed dependence of the flux with the size and the size distribution. Note that the $40 \mathrm{Mm}^{2}$ size corresponds to the $3 \times 10^{19}$ Mx flux.

I find that the magnetic flux $|\Phi|$ in each structure is very dependent on the size of the structures with a slope larger than one. This is to be compared to the results obtained by Harvey (1993) for large active regions ( $A$ above $373 \mathrm{Mm}^{2}$ ), who found a slope of 1 (i.e. $|\Phi|$ is proportionnal to $A$ ). The general behavior is not very different, especially for large active regions: For example she found a flux of $1.2 \times 10^{21} \mathrm{Mx}$ for a size of $800 \mathrm{Mm}^{2}$, which is close to the results presented here. However, an extrapolation at $A=1$ of Harvey's results gives $1.5 \times 10^{18} \mathrm{Mx}$ while we find smaller fluxes $\left(4 \times 10^{17} \mathrm{Mx}\right)$. Schrijver et al. (1996) also observed that the active region size is correlated 

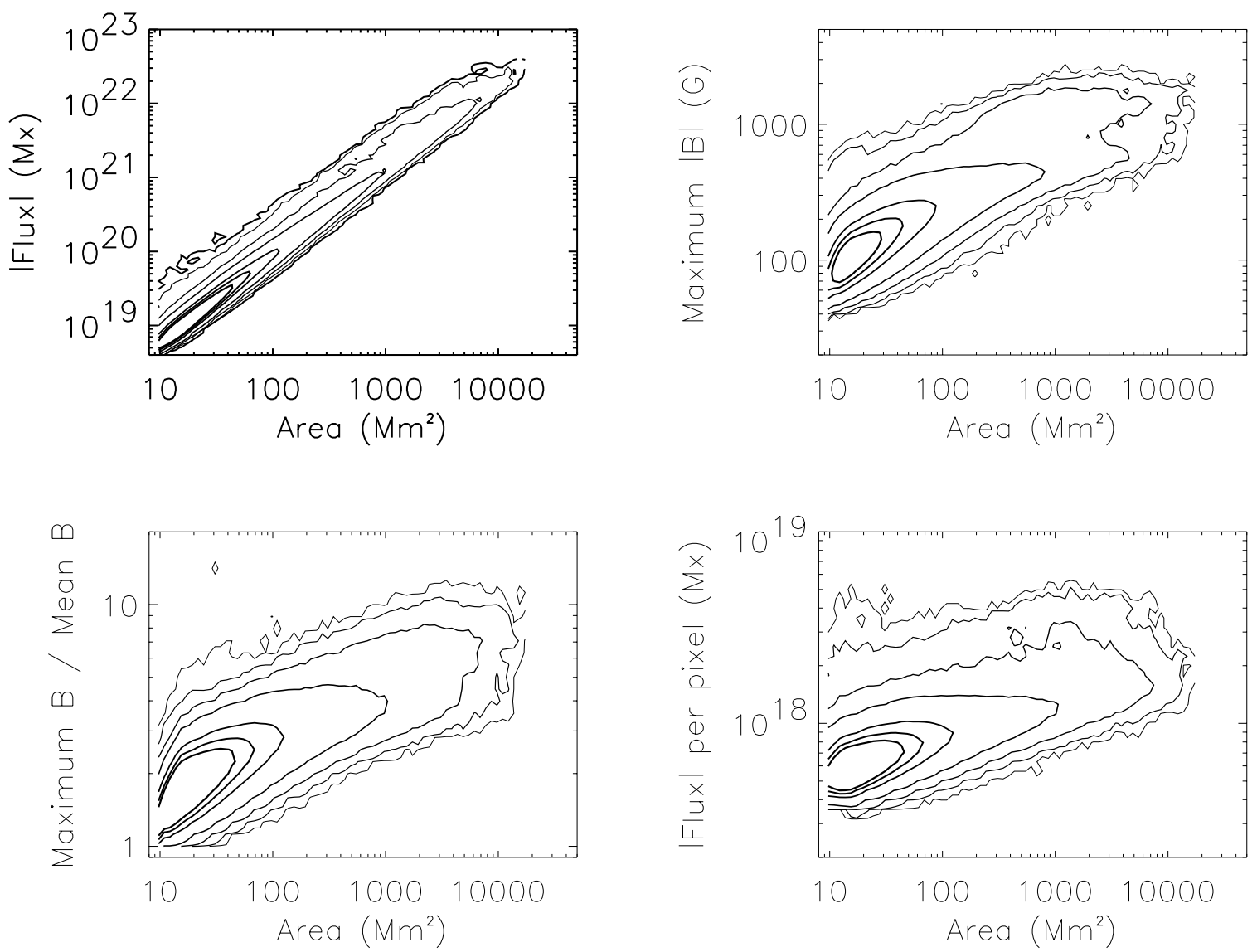

Fig. 5. Distribution of magnetic flux in all structures (resp. maximum magnetic field, ratio between maximum magnetic field and the average magnetic field over the structure and the magnetic flux density in the the structures) versus the size of the structures, for the $40 \mathrm{G}$ threshold. The lines correspond to $1,10,100,10^{3}, 10^{4}, 2 \times 10^{4}$ and $3 \times 10^{4}$ structures (from the outside to the center of each distribution).

with the mean magnetic flux in a region over a small field-ofview, but it concerns only a small range of sizes as well. The present study extends this result down to network structures and does not show any sharp transition between active regions and network structures (the same is true for the size distribution): although the properties are changing through the scales it is a gradual process over the three orders of magnitude in size.

Furthermore, the slope larger than 1 implies that the flux density $|\Phi| / A$ is also scale-dependent. The other parameters $\left(\left|B_{m}\right|\right.$ and $\left.B_{m} / \bar{B}\right)$ are also clearly dependent on the size but the slopes are much smaller than for the flux (see Table 3 ). The flux density in particular increases with the size but not by a large factor, as shown by the slope much smaller than $1(0.16-$ 0.18 ). We know that this parameter is either related to the filling factor or to the magnetic field strength in individual flux tubes constituting the region. From studies of contrast differences between network and faculae, Ortiz et al. (2002) found that faculae were constituted of larger flux tubes than the network. It is too early to make a direct comparison with the present results, especially because definitions of the network and faculae are different: They use the pixel magnetic field value to decided whether this is a network or facula pixel, while I do consider the size of the structures. More work need to be done to improve the comparison. However, the increase of flux density as a function of the size (factor of 2.8 between $10 \mathrm{Mm}^{2}$
Table 3. Slope of the $\log ($ parameter $)$ versus $\log (A)$ after averaging over small ranges of size $(\triangle \log A=0.1)$.

\begin{tabular}{ccc}
\hline \hline Parameter & $25 \mathrm{G}$ & $40 \mathrm{G}$ \\
\hline$|\Phi|$ & $1.210 \pm 0.007$ & $1.175 \pm 0.006$ \\
$\left|B_{m}\right|$ & $0.429 \pm 0.009$ & $0.384 \pm 0.007$ \\
$B_{m} / \bar{B}$ & $0.222 \pm 0.005$ & $0.209 \pm 0.004$ \\
$|\Phi| / A$ & $0.184 \pm 0.006$ & $0.161 \pm 0.005$ \\
\hline
\end{tabular}

and $10000 \mathrm{Mm}^{2}$ structures) may indeed be related to this contrast variabilities. The flux density also exhibits a large dispersion for a given size however, between $2 \times 10^{17}$ and $5 \times 10^{18}$ $\mathrm{Mx}$ (more than one order of magnitude), and this large dispersion is observed from small network features to active regions. There is a small departure from the power law (at small and large scales). The departure from the power law at very large scales for $\left|B_{m}\right|$ and $|\Phi| / A$ could be due to the non-linearity in MDI magnetic fields described in Sect. 2.3.3.

\subsection{Latitudinal and longitudinal distributions}

Synoptic maps for structures of different sizes show that small size structures (even below $40 \mathrm{Mm}^{2}$ ) are preferentially located around or at the location of active regions. This has already been observed for bipolar regions by Harvey (1993). 

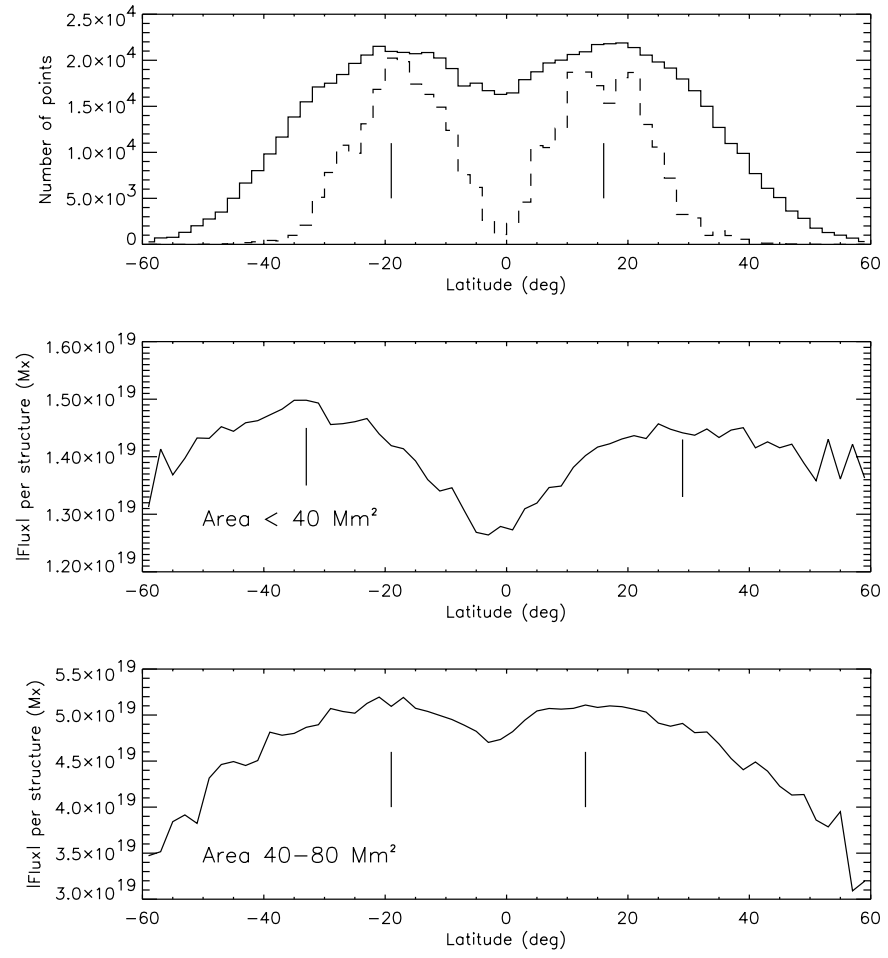

Fig. 6. Top: number of structures in a longitude range $\left[-10^{\circ}: 10^{\circ}\right]$ for area below $40 \mathrm{Mm}^{2}$ (solid line) and above $800 \mathrm{Mm}^{2}$ (dashed line, curve multiplied by 7) as a function of latitude. Middle: Average flux per structure (area below $40 \mathrm{Mm}^{2}$ ) as a function of latitude. Bottom: same but for structures in the range $40-80 \mathrm{Mm}^{2}$. The small vertical lines indicate the approximate position of the curve maxima.

Surprisingly, it appears to be true for network features as well: latitudinal distributions are shown in Fig. 6 (for the $40 \mathrm{G}$ threshold). Results are similar for the $25 \mathrm{G}$ threshold. The number of small structures (below $40 \mathrm{Mm}^{2}$ ) peaks at the same latitude within $2^{\circ}$ than active region number (as in Harvey 1993, for bipoles), around latitudes $-19^{\circ}$ and $15^{\circ}$ for the whole time period studied here.

On the other hand, the latitudinal variation of the average flux in these small structures have a maximum around latitudes $-33^{\circ}$ and $29^{\circ}$ as shown in Fig. 6. Only structures in the longitude range $\left[-10^{\circ}: 10^{\circ}\right]$ are selected, i.e. close to the central meridian (however, results are similar when all longitudes are considered). The average flux per structures is the largest in the northern hemisphere (respectively the southern hemisphere) is $11 \%$ (respectively $9.5 \%$ ) larger than at the equator, with a value close to $1.5 \times 10^{19} \mathrm{Mx}$. At high latitudes $\left(60^{\circ}\right)$ the flux is decreasing again toward values similar to those at the equator. This means that the small structures have their largest magnetic flux at a latitude larger (by $\sim 10^{\circ}$ ) than the latitude where their number is maximum. At these positions the number of detected structures has decreased by $18 \%$ in the North $(27 \%$ in the South) compared to the maximum number. However, the exact values could be slightly biased by projection effects.

The stronger concentration of flux at higher latitudes in small structures may be due to a combination of two processes: (i) the poleward meridional circulation in surface observed by many authors (see for example Meunier 1999b, and references therein), should transport preferentially the flux toward high latitudes; (ii) supergranulation may concentrate the flux in smaller structures compared to larger size structures at high latitude. Note that the the size distributions in different latitude ranges show an excess of small structures (below $40 \mathrm{Mm}^{2}$ ) compared to larger structures in the latitude range $30^{\circ}-40^{\circ}$ compared to the $20^{\circ}-30^{\circ}$ range.

The distribution of the small structures extends further toward large latitudes from the active region belt limit in the North $\left(\sim 14^{\circ}\right)$ than in the South $\left(\sim 9^{\circ}\right)$ at solar maximum. The smaller maximum $<|\Phi|>$ in the northern hemisphere (for $A<$ $40 \mathrm{Mm}^{2}$ ), could be due to a smaller concentration of flux per unit area in this hemisphere. On the other hand, one should remember that the northern hemisphere was more active during that period (Table 2). Therefore the effect at the origin of this North-South asymmetry must be strong. The NorthSouth asymmetries could be due an asymmetry of any of the process involved (supergranular diffusion, meridional circulation). However, results obtained by Meunier (1999b) suggests a weaker meridional circulation in the northern hemisphere though, while no study of asymmetry in the diffusion coefficient has been done. Instrumental effects, such as the larger noise in the South, may also play a role however. A detailed modeling of the different effects should therefore be done in order to understand better the observed asymmetries.

\subsection{Magnetic field environnement dependence}

\subsubsection{Immediate surrounding}

In a first approach I study the variation of the magnetic field characteristics when the activity level in a ring surrounding the structure is changing. I characterize this activity level in the neighborhood of the structure by the parameter $\alpha$, defined as the proportion of pixels above the magnetic threshold (i.e. $25 \mathrm{G}$ or $40 \mathrm{G}$ ) in the region $[R+20, R+40]$ pixels (see Sect. 2.2). A distance of 30 pixels at disk center corresponds to $42 \mathrm{Mm}$. $\alpha$ takes values between 0 and $97 \%$ and is linear in the range $5-65 \%$ on a linear-log scale. Results above $70 \%$ are not significant due to the smaller number of points.

When considering a sample of small magnetic features only (with a size below $20 \mathrm{Mm}^{2}$ ), the average size is decreasing by $2 \%$ when $\alpha$ is increasing (i.e. when the activity surrounding the feature is increasing) from 5\% to $65 \%$ : therefore the size distribution does not change very much. For structures with $A<40 \mathrm{Mm}^{2}$, the average size is quite constant also. However the average flux is meanwhile increasing by $28 \%$ and the flux density by $26 \%$, for the $40 \mathrm{G}$ threshold and $A<20 \mathrm{Mm}^{2}$ (respectively $37 \%$ and $35 \%$ for $A<40 \mathrm{Mm}^{2}$ ). Results are similar for the $25 \mathrm{G}$ threshold. Given the dependence of $\Phi$ versus $A$ and the small decrease in $A$, the flux is expected to decrease slightly if the properties of the network structures were constant. Therefore small features of a given size seems to have significantly stronger magnetic fluxes when their immediate surrounding is more active. This is likely to be related to the diffusion of magnetic flux from active regions to the network and the decay into network patches such as those observed here. 
Considering features of all sizes, I compute the slope of the magnetic flux $\Phi$ versus $A$ (log/log scale) as in Sect. 3.2, as well as the slope of the magnetic flux density versus $A$. For $\Phi$ versus $A$, the slope is increasing from 1.08 to 1.28 for $\alpha$ increasing from $5 \%$ to $65 \%$. Given the values obtained at $A=1$ from this fit, this means that the larger the size is, the larger the increase in flux is when $\alpha$ is increasing. The same is true for the flux density.

\subsubsection{Proximity of active regions}

Another approach consists in computing the minimum distance $\delta$ to regions larger than $800 \mathrm{Mm}^{2}$ in order to look at larger scales. Projection effects are taken into account when computing this distance. To avoid biases when a structure is far from disk center (meaning that its whole surrounding is not visible), only features in a box of $200 \times 200$ pixels at disk center are considered (latitudes and longitudes are then in the range $\left.\pm 24^{\circ}\right)$. For $11 \%$ of them there is no large structure on the disk (this happens mostly during cycle minimum). $\delta$ takes values between $0.1^{\circ}$ and $72^{\circ}$. The distribution of $\delta$ shows a maximum around $5^{\circ}$ and then decreases sharply, especially above $55^{\circ}$. The shape of the distribution is however slightly different depending on the cycle phase (flatter at cycle minimum, sharper decrease at cycle maximum). A value of $\delta$ of $10^{\circ}$ corresponds to $121 \mathrm{Mm}$.

When looking at small magnetic structures (below $20 \mathrm{Mm}^{2}$ ), I find that although the average size is remaining constant, the average flux is decreasing linearly by $11 \%$ when $\delta$ increases from $2.5^{\circ}$ to $42.5^{\circ}$. The flux density is also decreasing, by $10 \%$. For $A<40 \mathrm{Mm}^{2}$, results are similar although of larger amplitude, with percentages of $22 \%$ and $13 \%$ respectively. The results are similar for the $25 \mathrm{G}$ threshold. When differentiating for the 3 phases of the cycle defined in Sect. 2.1, it is found that the variation is mostly observed at cycle minimum and during the ascending phase: there is a sharp decrease up to $\delta=10^{\circ}$, then the decrease is much slower. The decrease is of $13 \%$ during the minimum and of $8 \%$ during the ascending phase. During cycle maximum, there is almost no variation.

Therefore, results are qualitatively similar to the previous analysis but at a larger scale: The flux in small magnetic structures of a given size is decreasing when the structure is further from an activity center. The results for the $11 \%$ with no structures larger than $800 \mathrm{Mm}^{2}$ on the disk confirm this. These network properties are consistent with the argument of Schrijver et al. (1997) that each time there is cancellation of network structures (of opposite polarities), only the residual (because of a non-zero flux imbalance) will reappear again. Therefore we expect less flux to concentrate far from active region location for availability reasons. A given quiet or enhanced network region could also be related to a strong activity happening at the same location a long time before (several rotations for example).

On the other hand the slope of the flux versus the size (for structure of all sizes) does not show any clear behavior. The same is true for the flux density. This mean that the flux variation is similar for all scales.

\subsection{Bipolar structures}

I extract a subset of structures by selecting bipoles using the rules defined by Hagenaar (2001): the maximum distance between structures is set to $10 \mathrm{Mm}^{2}$ and the difference in flux is smaller than $33 \%$. This analysis provides a subset of 29337 bipolar structures. Although this subsample does not correspond exactly to small ephemeral regions (because of possible chance encounters between network features of opposite polarity), the proportion of ephemeral regions in this subsample is likely to be much larger than in the general sample, allowing to compare qualitatively network and ephemeral region properties. An exhaustive analysis of ephemeral regions is outside the scope of this paper (see Hagenaar et al. 2003, for such a study). The slopes of the parameters defined in Table 3 for the whole data set are larger for this selection of bipoles. When considering a subsample of structures with sizes below $40 \mathrm{Mm}^{2}$ only, $\Phi$ is larger by $3.7 \%$ in bipolar structures compared to features which are not involved in a bipolar structure, $\left|B_{m}\right|$ is larger by $6.7 \%, B_{m} / \bar{B}$ is smaller by $1.3 \%$ while $\Phi / A$ is larger by $7.4 \%$ (for the $40 \mathrm{G}$ threshold). Note that the average size is also smaller (by 3.5\%). Results are similar for the $25 \mathrm{G}$ threshold. Therefore small features not involved in bipoles do not have the same magnetic characteristics than bipoles of similar size.

\section{Solar cycle variations}

\subsection{Size distribution}

The average number of structures of all sizes per image is increasing from 237 at cycle minimum to 864 at cycle maximum (for the $40 \mathrm{G}$ threshold). Figure 7 shows the size distribution for the three periods of the cycle defined in Fig. 1. It was, of course, expected to have a much larger number of large structures during cycle maximum. This is true for all sizes, confirming the recent results about the correlation between network variations and the solar cycle. The shape of the distribution is quite similar although the slope is different in the size range of the distribution where a power law is observed (smaller at cycle maximum) and this power law regime seems to extend toward larger sizes at cycle maximum. This result is therefore different from that of Harvey (1993), who found that the shape of the size distribution is an invariant with time. The difference could have been due to the fact that, in this work, more weight is given to structures with a longer lifetime: a longer lifetime of large active regions at cycle maximum could explain the result. However, she found the opposite (faster decay rate at cycle maximum).

\subsubsection{Number of structures of various sizes and fluxes}

The variation of the number of structures versus time for different ranges in magnetic flux $|\Phi|$ is shown in Fig. 8 for the $40 \mathrm{G}$ threshold. Results are similar for the $25 \mathrm{G}$ threshold and for different ranges in size. I find that, at first order, structures of all types exhibit a similar increase with the solar activity level, with mostly a difference in amplitude.

I also observe that the increase at the beginning of the ascending phase seems to happen first for large active 


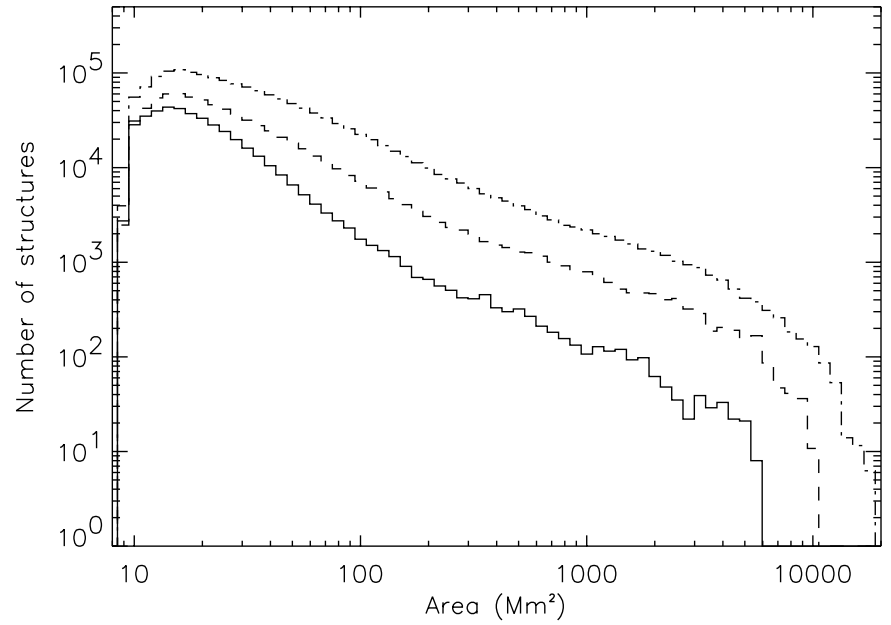

Fig. 7. Size distribution during the three phases of the cycle: cycle minimum (solid), ascending phase (dashed) and cycle maximum (dotted-dashed), for the $40 \mathrm{G}$ threshold.

regions (flux above $10^{21} \mathrm{Mx}$, corresponding to areas larger than $800 \mathrm{Mm}^{2}$ ) and medium size regions (in correlation with the Wolf number), and then later for the smaller structures (below $40 \mathrm{Mm}^{2}$ ). The difference is of the order of one of our 100day period; this corresponds to our sampling, therefore it would be useful to use a smaller time step to check this result. This is a difficult task, however, because of the data gaps and this should be investigated further using another data set. This result seems in contradiction with the observations made by Harvey (1993) that the minimum of the cycle occurs one year before for small bipolar structures compared to large ones. Our sample is dominated by non-bipolar structures however, which may explain the discrepancy if much of the network - which is dominating the sample - is fed by decaying active regions. Later during the cycle, the variation for these three types of structures seems similar although again not in phase.

The amplitude of the variation (illustrated in Fig. 8, bottom) is of course very large for large structures (factor of 15.2 in number for example for flux above $10^{21} \mathrm{Mx}$, and factor of 19.6 in integrated flux), and much smaller for small structures (factor of 2.9 in number for flux below $3 \times 10^{19} \mathrm{Mx}$, and factor of 3.5 in integrated flux). The ratio between the number of structures at maximum and minimum is therefore increasing with increasing size or magnetic flux in the structure. During solar maximum the Sun is more largely covered by strong (and large) magnetic structures, which leaves less "free area" for the small magnetic structures. However, if we correct the variations for small features by dividing the numbers by the "free area", the results are not modified significantly. Foukal et al. (1991) found a ratio of 1.5 between maximum and minimum for network features. Typical network patches corresponding to fluxes below $\sim 5 \times 10^{19}$ Mx approximately, and Fig. 8 shows that the variation may be of larger amplitude for the network compared to what have been found by Foukal et al. (1991), close to a factor 7 instead of 1.5. We also find a larger factor ( 7.9 for bipolar regions smaller than $373 \mathrm{Mm}^{2}$ ) than Harvey's (1993) results for ephemeral regions, who found a ratio of 2-2.5. Surprisingly, while results of the past decade tended to show that the network
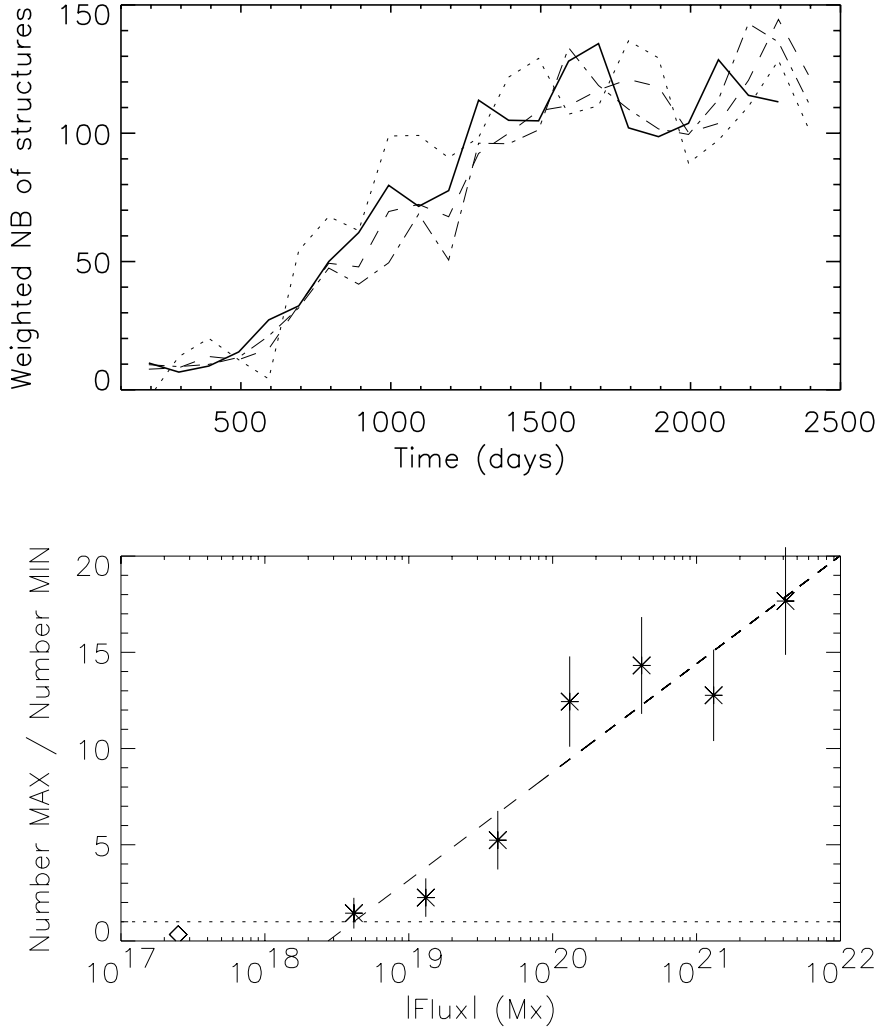

Fig. 8. Top: variation of the weighted number of structures for fluxes smaller than $3 \times 10^{19} \mathrm{Mx}$ (dotted), between $3 \times 10^{19}$ and $10^{21} \mathrm{Mx}$ (dashed) and above $10^{21} \mathrm{Mx}$ (dotted-dashed); the thick solid line is the Wolf number. Curves are normalized to have the same average number during cycle minimum and during cycle maximum. Numbers are also normalized to the number of images in the period. Bottom: The stars represent the ratio between the number of structures at activity maximum and minimum for different ranges of flux (range of $\log \Phi=0.5$ ), and the dashed line is a linear fit over these points (in the log-linear plot. The horizontal dotted line shows the ratio $=1$ level (reversal of the variation during the solar cycle) and the diamond point represent the result of Muller \& Roudier (1984) assuming a flux of $2.5 \times 10^{17}$ Mx per individual NBP.

variations were anti-correlated with the solar cycle, a very recent study by Hagenaar et al. (2003) using an approach close to the present one found a weak anti-correlation of the number density of emerging ephemeral resgions, also observed for the network. This discrepancy is not yet understood.

An extrapolation shows that this ratio would reach 1 (below which the variation with time would be anti-correlated with the solar cycle) very close to the lower boundary of the range studied here (between 1 and $10 \mathrm{Mm}^{2}$ and close to $10^{18} \mathrm{Mx}$ ). The departure from the linear fit (in the log-linear plot) is only at the $1-\sigma$ level. This extrapolation suggests that there could be a reversal of the variation of the flux in small structures with solar activity compared to larger structures such as studied in this paper. This could be related to the observations made by Muller \& Roudier $(1984,1994)$ that the number of NBP observed at very high spatial resolution (in the $G$-band) in the quietest zones available is anti-correlated with the solar cycle. 


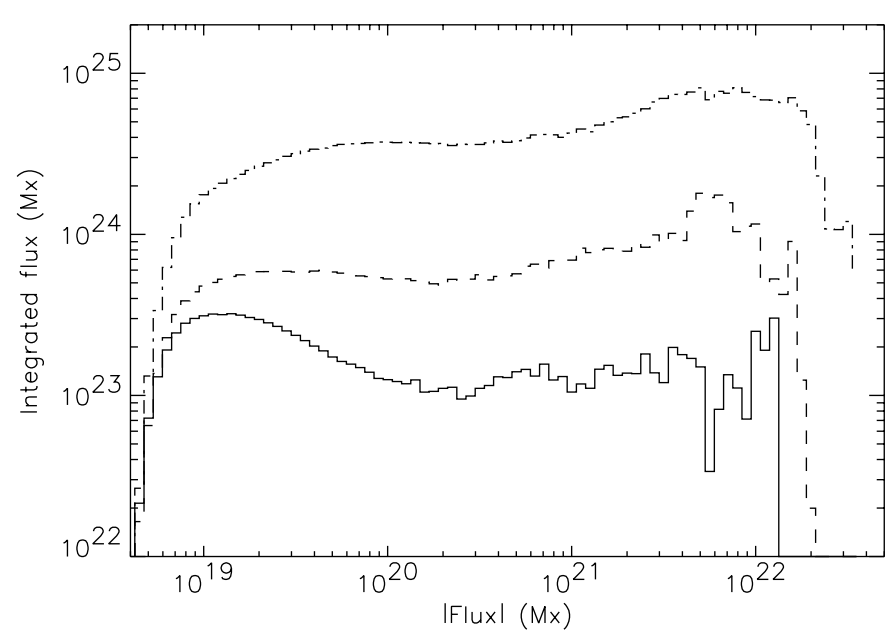

Fig. 9. Magnetic flux distribution during the three phases of the cycle: cycle minimum (solid), ascending phase (dashed) and cycle maximum (dotted-dashed), for the $40 \mathrm{G}$ threshold.

\subsubsection{Magnetic flux distribution}

Another interesting parameter is the integrated magnetic flux for a given range in size or magnetic flux. As expected from Fig. 8, we find an increase of the total flux for the three ranges of flux (below $3 \times 10^{19} \mathrm{Mx}$, above $10^{21} \mathrm{Mx}$ and in between). At the $40 \mathrm{G}$ threshold, the ratio is 0.96 at solar minimum (meaning that there is as much flux in structures with flux below $3 \times 10^{19} \mathrm{Mx}$ and above $10^{21} \mathrm{Mx}$ ) and 5.21 at solar maximum. The distribution of integrated flux versus the flux in the structures is shown in Fig. 9 for the three periods (cycle minimum, ascending phase and maximum): The flux in network structures is predominant during solar minimum and the increase in flux in network structures is quite large along the cycle.

\subsection{Magnetic field characteristics}

The variation of the flux distribution during the solar cycle is similar to that of the size distribution shown in the previous section. Figure 10 shows the flux distribution variation for small structures only (below $40 \mathrm{Mm}^{2}$ ). I find that the distribution is extending toward larger fluxes from cycle minimum to cycle maximum (longer tail) and the maximum of the distribution is also shifted toward larger fluxes: The average magnetic flux $|\Phi|$ for $A$ lower than $40 \mathrm{Mm}^{2}$ increases by $26 \%$ from minimum to maximum. This is consistent with the larger variation found previously for the flux than for the number of structures (20\% expected). The other magnetic characteristics are also increasing: $33 \%$ for $\left|B_{m}\right|, 16 \%$ for $B_{m} / \bar{B}$, and $14 \%$ for the $|\Phi| / A$ (for the $40 \mathrm{G}$ threshold). The increase for $A<20 \mathrm{Mm}^{2}$ is slightly smaller as in the range $40-80 \mathrm{Mm}^{2}$. Values are similar or slightly larger in the range $40-400 \mathrm{Mm}^{2}$. In Sect. 3.4.2, I found that the average magnetic flux $|\Phi|$ was decreasing when the distance to an active region was increasing. One should note that curves for the different periods reflected the larger concentration of flux during cycle maximum, and that this is true for all distance to active region: in other words, at a given distance from an active region and for a given size, the average magnetic flux is larger at cycle maximum, the effect being stronger far

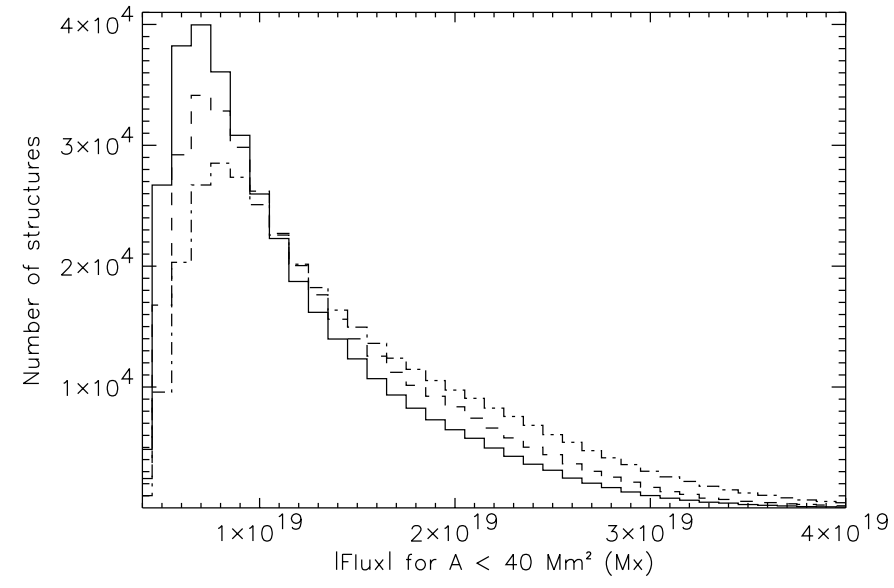

Fig. 10. Flux distribution for small magnetic features (below $40 \mathrm{Mm}^{2}$ ), during the three phases of the cycle: cycle minimum (solid), ascending phase (dashed) and cycle maximum (dotted-dashed), for the $40 \mathrm{G}$ threshold.

from active region. For very small structures (below $20 \mathrm{Mm}^{2}$ ), the variations for the subset of bipoles defined in Sect. 3.4 are much smaller than the variations for non-bipolar structures.

The slopes defined in Sect. 3.2 and Table 3 (magnetic characteristics versus size) are found to decrease along the solar cycle, while the values extrapolated for $A=1$ (from power law fits) are increasing. This is true for both magnetic thresholds. As a consequence, all parameters increase with the activity level for a given size, but the variation is more pronounced for small structures. The variation of the slope for the bipole selection is not as large however. This suggests that the processes leading to magnetic flux concentration in network patches is more efficient during solar maximum, but the dynamo process at the origin of the ephemeral regions of similar size (Schrijver et al. 1997) is not modified by a large factor. The observed variations for network features could also be due to the fact that more flux is available during solar maximum; however the link is not direct because more flux means also more cancellations since opposite polarity features meet more easily (see Harvey 1993, showing that magnetic flux from active region was removed in 4 months at solar cycle maximum and 10 months at solar cycle minimum for this reason).

The observed variation along the solar cycle could be due to a larger availability of magnetic flux, but it could also be related to the relationship between network components and granulation or supergranulation. Several processes could lead to such a variation: (i) Size of intersupergranule. However Singh \& Bappu (1981) found no variation with the solar cycle. (ii) Horizontal velocities in granules and supergranules. (iii) Number of explosive granules during the cycle. They are an important factor if they are at the origin of supergranular flows (Rieutord et al. 2000). (iv) Diffusion coefficient. (v) Supergranular cell sizes. Münzer et al. (1989) found an increase of cell size with the activity level, while Singh \& Bappu (1981) found the opposite using data obtained at several maximum and minima. More recently, Berrilli et al. (1999) also found an increase of supergranular cell sizes with the solar activity level using 1 year of data. We know that magnetic elements 
are confined along the edges of supergranules, and those which emerge inside the cells are quickly advected toward the edges (Lisle et al. 2000). If the cells are smaller at cycle maximum, there will be less space available at the borders of supergranules (perimeters of the cells) and therefore we could expect a given amount of flux to be more concentrated. On the other hand, if the cells are smaller we could expect a larger cancellation rate. I am not aware of studies about points (ii), (iii), and (iv). Concerning point (iv), we know however that an increased amount of flux during the solar maximum is diffusing into the network. A modeling of these processes could be useful to determine whether the larger flux concentrations is only due to the increase of available flux at cycle maximum or to additionnal processes. Such a modelisation is beyond the scope of this paper. Models by Schrijver (2001) and Parnell (2002) would be adequate to study the influence of these parameters.

The more concentrated magnetic flux in structures of small sizes such as network patches during solar maximum may explain the observation of Muller \& Roudier (1984) mentionned in the previous section: There may be less flux in the quietest regions selected in these observations due to a larger concentration in others. A complementary result has been obtained by Dermendjev et al. (1994), studying the clusters of NBP using high resolution observations in the $G$-band. They used the same data set than Muller \& Roudier (1984), i.e. there may be a bias in the selection of the quietest region on the Sun at a given time. They found a predominence for smaller clusters (5.2 arcsec) with more NBP (typically 4 NBP per clusters) during minimum, while at maximum they found a predominence for larger clusters (6.5 arcsec) with less NBP per clusters (two): This result seems to be in contradiction with the observations presented in this paper showing that magnetic flux seems to be more concentrated at solar maximum. This could be due to a selection effect, because the quietest regions are not necessarily representative of the whole surface. Note also that the anti-correlation of NBP numbers with the solar cycle has often been related to the anti-correlation between $\mathrm{X}$ bright points and the solar cycle. However this latter variation may be an artifact of the analysis as shown by Sattarov et al. (2002).

\section{Summary}

The study of the properties of magnetic features over more than 3 orders of magnitude in size (ranging from the network to active regions) during the first half of solar cycle 23 using an extensive data set of low-noise full disk magnetograms led to the main following new results.

1. The size and flux distributions exhibit 3 main domains: The range below $40 \mathrm{Mm}^{2}\left(3 \times 10^{19} \mathrm{Mx}\right)$, the range $40-400 \mathrm{Mm}^{2}$ $\left(3 \times 10^{19}-10^{21} \mathrm{Mx}\right)$ where the distributions are power laws, and the range above $400-700 \mathrm{Mm}^{2}\left(\sim 10^{21} \mathrm{Mx}\right)$. This latter typical scale, close to the supergranular scale, is found to increase from cycle minimum to solar maximum. The shape of the size distribution is not exactly an invariant along the solar cycle.

2. A strong power law dependence of the magnetic flux of the structures on their size (from small network patches to active regions) suggests continuity from small scales to large scales. The slope larger than 1 means that the flux density is also size-dependent. The size-dependence of the magnetic flux is less pronounced during solar maximum however: Differences between solar minimum and maximum are more pronounced for small structures. The magnetic flux of small structures is increasing from solar minimum to solar maximum (for a given area), suggesting that there are more flux tubes concentrated in the same area or the intrinsic magnetic field in the flux tubes is larger at cycle maximum. Small structures involved in a bipolar configuration present larger magnetic fluxes (as well as larger maximum magnetic fields and flux densities) for very small sizes, but their variation along the solar cycle is less pronounced than for other features of similar size.

3. The maximum magnetic field, the ratio between maximum and average magnetic field and the flux density exhibit a behavior similar to the magnetic flux although the dependence on the size is less pronounced. In addition I observe a very large dispersion of the flux density for structures of a given size. The average values of these parameters for a given size are also increasing with increasing solar activity.

4. The North-South asymmetry of the number of structure is reversing at small sizes for the $25 \mathrm{G}$ threshold only (below $40 \mathrm{Mm}^{2}$ ), suggesting a different origin of these weakest flux structures. The North-South asymmetries for the two polarities is compatible with the fact that features of leading polarity are easier to detect (as is expected for active regions) for structures of all sizes including the network structures. The global flux imbalance is not understood.

5. The East-West asymmetry is compatible with an eastward inclination of flux tubes, including for network features although they result from the decay of active region and do not correspond to newly emergent magnetic flux. The East-West asymmetry is slightly different for leading and following polarities (although of the same sign), which could be due to a relative inclination between the two polarities with respect to each other in addition to the global eastward inclination.

6. There is a strong dependence of the magnetic flux of small structures with the activity of their surrounding, both at small scale (immediate surrounding) and large scale (distance from a large active region): Their flux, as well as their flux density, are decreasing when they are further from an active region. At small scales, the effect is of larger amplitude for active regions compared to smaller structures.

7. The latitudinal distribution of structures of various sizes as well as the butterfly diagrams confirm that small structures appear in larger number in the region of high activity level. However the flux per structure in small features (below $40 \mathrm{Mm}^{2}$ ) is maximal at higher latitudes. A North-South asymmetry is also observed.

8. I observe a similar variation of the integrated flux for various size or flux ranges with time, except for the amplitude which is smaller for small and weak flux structures, i.e. the number of structures is correlated with the solar cycle for all sizes. The amplitude of network variations appears to be larger than what have been found previously however (factor 7). A time lag of the order of 100 days (between regions with size above $40 \mathrm{Mm}^{2}$ and below) is observed but must be investigated further. 
9. The amount of flux at cycle minimum is of the same order of magnitude in large active regions and in network structures.

\section{Conclusion}

Our results suggest that the properties of structures from active regions down to network features are varying continuously. However we have identified a typical scale corresponding to the supergranular scale using different approaches, which is increasing from cycle minimum to cycle maximum. Structures above this size tend to behave differently than smaller structures, no transition being observed between network structures and small regions. This suggests a modification of the properties between small and large active regions - at the supergranular scale - and not only between active regions and network structures. The variation of that scale along the solar cycle is compatible with recent results showing that the supergranular cells are increasing from solar minimum to solar maximum, which would suggest that this typical scale may indeed by related to supergranulation cells or to the processes at the origin of supergranulation.

It is interesting to consider the different behavior of small and large scales structures concerning the slope of $|\Phi|$ (or the other magnetic properties characteristics) depending on the approach: The slope of the magnetic flux versus size is decreasing when the activity level is increasing along the solar cycle while it is increasing when the local activity level is increasing. On the other hand, no significant variation is observed when looking at these slopes for different distances to a large active region. This shows that although the flux is always larger (at a given size) when it is "more active", this variation is different for the various sizes. Variations observed for network structures may be due to a variation of granulation or supergranulation properties, while the variation for active regions and ephemeral regions is more likely to be due to the emergence of magnetic field due to the dynamo processes, including a possible variation at the bottom of the convective zone and a strong relation between the involvement of the region in a nest and its characteristics.

For the largest structures, the main result is that active regions have different properties when they are close to another one: Not only active regions seem to emerge preferentially in regions previously occupied by other active regions as observed by other authors (the presence of active longitudes or nests has been observed by many authors, for example Gaizauskas et al. 1983; Harvey \& Zwaan 1993; Harvey 1993; Schrijver \& Zwaan 2000), but the flux is more concentated in these structures than for isolated ones: we do not identify strictly the nests, however active regions close to another one are more likely to be part of a nest. Harvey (1993) has suggested from a small sample that the properties of active regions emerging in a preexisting spot region is related to the already emerged flux, by observing the orientation of bipoles. We confirm this by using a different approach: The flux characteristics of a structure seems influenced by the involvement in a nest or not. The larger flux at cycle maximum could be related to this, because she observed that the emergence rate in nests was larger during solar maximum $(50 \%)$ than during solar minimum $(30 \%)$. When looking at small-scale behavior (close environment), the large regions are more sensitive to their close environment compared to small features.

The small bipolar structures however, related to ephemeral regions, have properties different from network features of the same size. Although for all structures the magnetic flux (as well as other magnetic characteristics) is larger when the environment is more active or during cycle maximum, the detailed variation of the concentration of the magnetic field in network structures is different from that of small bipolar structures. This difference is also likely to be due the difference in origin: Creation by the dynamo process (similar to large active regions) and decay from active regions. Results suggest that surface motions (such as in granules and supergranules) are less efficient to concentrate the flux than the dynamo process but exhibits larger variations along the solar cycle. However part of the bipolar association are probably due to network patches resulting from the decay of some active region just before they merge to participate in the flux removal processes, so the differences are probably underestimated.

The fact that the average magnetic flux of small structures is not the largest at latitudes where activity occurs is not necessarily inconsistent with the observation that the flux is decreasing when the structures are further from an active region. The latter approach averages the dependence in all directions. This suggests that the diffusion of magnetic flux is not isotropic, possibly due to the meridional flows that are predominantly poleward. A possible interpretation for the observed latitudinal variation could be the following: At latitude of strong activity, supergranular diffusion may not be very efficient so that the concentration of small patches of flux are weaker; at high latitude, the supergranulation may be more effective in concentrating the flux, available in larger quantity than close to the equator due to the meridional circulation. However it is surprising that this is not true for structures in the range $40-80 \mathrm{Mm}^{2}$ also, which are also network structures resulting from the flux dispersal at the surface. The small number of features close to the equator is indeed surprising because this is also where the detection is the easiest.

We suggest that the anti-correlation of NPB with the solar cycle found by Muller \& Roudier (1984) may be due to both a selection effect (the quiestest regions at a given time) and the largest concentration of the flux in network patches during solar maximum. The variation of the magnetic properties of the network structures may also be related to the different contrasts of network and plages found by Ortiz et al. (2002). These results are therefore very important in the context of the solar irradiance variations. Furthermore, the contribution of the magnetic network is probably larger than what have been previously observed.

In the future it would be very useful to perform simulations such as the ones performed by Simon et al. (2001) and Krijger $\&$ Roudier (2003) by including solar cycle variations of parameters such as the supergranulation size (see Sect. 4.2) in order to help to interpret these new results. It would also be interesting to compare whether global models such as those made by Schrijver (2001) and Schrijver et al. (2002) can reproduce such 
results. The model of the magnetic carpet elaborated by Parnell (2002) could also be used. These models reproduced with success the histogram of flux densities. They would also be useful to check the difference in efficiency between supergranular diffusion and meridional circulation in transporting the flux, as well as their solar cycle dependence, and their influence on the flux contents of structures of a given sizes.

More observationnal work will also be needed to confirm the new results obtained in this work, in particular by performing large field-of-view network observations ( $G$-band, magnetograms and dopplergrams) and spectropolarimetric measurements at very high spatial resolution. Both types of observations should be performed regularly during at least a solar cycle.

Acknowledgements. I am very grateful to L. van Driel for her careful reading of a preliminary version of the manuscript and many useful comments that helped to improve it. I also thank the anonymous referee for his/her comments on this paper. SOHO is a mission of international cooperation between the European Space Agency (ESA) and NASA. Sunspot numbers have been provided by the National Geophysical Data Center (National Oceanic and Atmospheric Administration).

\section{References}

Abbett, W. P., \& Fisher, G. H. 2001, ApJ, 546, 1194

Berger, T., \& Lites, A. 2003, Sol. Phys., 213, 213

Berrilli, F., Ermolli, I., Florio, A., \& Pietropaolo, E. 1999, A\&A, 344, 965

Caligari, P., Moreno-Insertis, F., \& Schüssler, M. 1995, ApJ, 441, 886

Dermendjev, V. N., Muller, R., \& Madjarska, M. S. 1994, Sol. Phys., 155,45

Foukal, P., \& Lean, J. 1988, ApJ, 328, 347

Foukal, P., Harvey, K., \& Hill, F. 1991, ApJ, 383, L89

Gaizauskas, V., Harvey, K. L., Harvey, J. W., \& Zwaan, C. 1983, ApJ, 265, 1056

Garcia de la Rosa, J. I. 1983, Sol. Phys., 89, 51

Hagenaar, H. J. 2001, ApJ, 555, 448

Hagenaar, H. J., Schrijver C. J., \& Title, A. M. 2003, ApJ, 584, 1107
Harvey, K., \& Zwaan, C. 1993, Sol. Phys., 148, 85

Harvey, K. L. 1993, Ph.D. Thesis, Astronomical Institute, Utrecht University

Howard, R. 1991, Sol. Phys., 134, 233

Krijger, J. M., \& Roudier, T. 2003, A\&A, 403, 715

Labonte, B. J., \& Howard, R. 1982, Sol. Phys. 80, 15

Lean, J. L., Cook, J., Marquette, W., \& Johannesson, A. 1998, ApJ, 492, 390

Lisle, J., De Rosa, M., \& Toomre, J. 2000, Sol. Phys., 197, 21

Meunier, N. 1999a, ApJ, 515, 801

Meunier, N. 1999b, ApJ, 527, 967

Muller, R., \& Roudier, T. 1984, Sol. Phys., 94, 33

Muller, R., \& Roudier, T. 1994, Sol. Phys., 152, 131

Münzer, H., Hanslmeier, A., Schröter, E. H., \& Wöhl, H. 1989, A\&A, 213,431

Nishikawa, J. 1994, Sol. Phys., 152, 125

Ortiz, A., Solanki, S. K., Domingo, V., Fligge, M., \& Sanahuja, B. 2002, A\&A, 388, 1036

Parnell, C. E. 2002, MNRAS, 335, 389

Sattarov, I., Pevtsov, A. A., Hojaev, A. S., Sherdonov, C. T. 2002, ApJ, 564,1042

Rieutord, M., Roudier, T., Malherbe, J.-M., \& Rincon, F. 2000, A\&A, 357,1063

Sànchez Almeida, J., \& Lites, B. W. 2000, ApJ, 532, 1215

Scherrer, P., Bogart, R. S., Bush R. I., et al. 1995, Sol. Phys., 162, 129

Schrijver, C. J., Shine, R. A., Hagenaar, H. J., et al., 1996, ApJ, 468, 921

Schrijver, C. J., Title, A. M., van Ballegooijen, A. A., Hagenaar, H. J., \& Shine, R. A. 1997, ApJ, 487, 424

Schrijver, C. J. 2001, ApJ, 547, 475

Schrijver, C. J., \& Zwaan, C. 2000, Solar and Stellar Magnetic Activity, Cambridge Astrophysics Ser. 34 (Cambridge Univ. Press)

Schrijver, C. J., De Rosa, M. L., \& Title, A. M. 2002, ApJ, 577, 1006

Sigwarth, M. 2001, ApJ, 563, 1031

Singh, J., \& Bappu, M. K. V. 1981, Sol. Phys., 71, 161

Simon, G. W., Title, A. M., \& Weiss, N. O. 2001, ApJ, 561, 427

Socas-Navarro, H., \& Sànchez Almeida, J. 2002, ApJ, 565, 1323

van Driel-Gesztelyi, L., \& Petrovay, K. 1990, Sol. Phys., 126, 285

Wang, H. 1988, Sol. Phys., 116, 1

White, O. R., \& Livingston, W. C. 1981, ApJ, 249, 798 